INFLAMMATORY BOWEL DISEASE

\title{
In siblings with similar genetic susceptibility for inflammatory bowel disease, smokers tend to develop Crohn's disease and non-smokers develop ulcerative colitis
}

\author{
S Bridger, J C W Lee, I Bjarnason, J E Lennard Jones, A J Macpherson
}

Gut 2002;51:21-25

See end of article for authors' affiliations

Correspondence to: Dr A J Macpherson Institute of Experimental Immunology, University Hospital Zürich,

Schmelzbergstrasse 12,

$\mathrm{CH}-8091$ Zürich,

Switzerland;

amacpher@pathol.unizh.ch

Accepted for publication 2 October 2001

\begin{abstract}
Background and aims: Smoking tobacco has opposite effects on the different forms of inflammatory bowel disease (IBD). It predisposes to the development of Crohn's disease (CD) yet is associated with a reduced incidence of ulcerative colitis (UC). We have studied sib pairs discordant for both smoking and IBD phenotype (UC or CD) to investigate whether smoking determines the type of IBD that develops in individuals with very similar genetic susceptibility.

Patients: Smoking habits and disease characteristics were analysed in 242 IBD pedigrees 1658 patients). Within this group there were 339 affected sibling pairs of whom 89 were discordant for smoking when diagnosed.

Results: Smoking at diagnosis was associated with development of CD (odds ratio (OR) 3.55; $95 \%$ confidence limits 2.50-5.02; $<<0.001$ ) in all of the familial patients, with increases when analysed for ileocaecal disease, fibrostenosis, and intestinal resection. Smokers were also protected from UC IOR $0.28 ; 0.2-0.4 ; \mathrm{p}<0.001$ ). Of 89 sibling pairs discordant for smoking at diagnosis, 23 were also discordant for disease type -in 21 of these, CD occurred in the smoker and UC in the non-smoker (OR $10.5 ; 2.6-92 ; \mathrm{p}<0.0001)$.

Conclusions: Smoking is a strong environmental risk factor for Crohn's disease and increases the likelihood of needing surgery. However, sib pairs who are discordant for both smoking and IBD type almost always show CD in the smoker and UC in the non-smoker, and so in some cases tobacco consumption acts on IBD genetic predisposition to shift the phenotype from UC towards CD. The explanation of part of the apparent "protective" effect of smoking on sporadic UC may be that the form of IBD that develops in a proportion of smokers is not UC but CD.
\end{abstract}

W ithin the broad grouping of inflammatory bowel disease (IBD), Crohn's disease (CD) and ulcerative colitis (UC) are perceived as distinct conditions, albeit as part of a clinical spectrum which can include intermediate or indeterminate variants. ${ }^{1}$ There is considerable evidence for this phenotypic distinction ${ }^{2}$ : the intestinal sites (CD potentially anywhere in the intestine, UC confined to the colon) and pathological features (CD granulomatous transmural inflammation, UC chronic mucosal inflammation) are different; the cytokine profiles characteristic of CD $\left(T_{h 1}\right)$ and UC immunopathogenesis are distinct ${ }^{3}{ }^{4}$; and treatments and their efficacy differ between the two conditions. These phenotypic distinctions have naturally led to an expectation of finding genes (or genetic linkages) and animal models that are specifically associated either with CD or UC.

The genetics of IBD are complex and polygenic. In humans, a large number of whole genome scans have reported multiple linked loci for $\mathrm{CD}$ and $\mathrm{UC}^{5-22}$ of which one locus contains the NOD2 gene which predisposes to CD. ${ }^{23-25}$ To identify all of the genes responsible for linkages and their relative contributions to the IBD phenotype will be a significant task. For this, it is important to understand, and where possible control for, the environmental factors that act on a particular genetic background to determine the disease that develops.

Smoking is an environmental factor that is comparatively easy to define and strongly associated with $\mathrm{CD}^{26}$ In contrast, epidemiological studies have repeatedly shown an inverse correlation between the prevalence of smoking and the incidence of UC. ${ }^{27}$ We have previously found in a study of first degree relatives in mixed pedigrees (which contain some members with CD and others with UC) that smokers are overall more likely to develop CD and non-smokers to develop UC. ${ }^{28}$ However, in this earlier study (which was focused on comparing the clinical features of familial and sporadic IBD) we looked at parent-child relationships in addition to sib pairs, and comparison of the relatively small number of sib pairs (13) discordant for both smoking and IBD type just failed to reach significance $(\mathrm{p}=0.06)$ for smoking as an environmental factor influencing the development of CD. Although the analysis with additional parent-child pairs was significant, ${ }^{28}$ the parent-child pairs grew up at different times and were subject to very different environmental, hygiene, and vaccination influences, in a period when the incidence of both CD and smoking was changing in the population as a whole; hence we were deliberately cautious in our interpretation of the result. Yet the effect is very important: it raises the possibility that smoking may act on a specific genetic background, where the risks between CD and UC are evenly balanced, to influence whether the outcome is CD in smokers or UC in non-smokers. In other words, some of the apparent protection that smoking exerts on sporadic UC might be due not only to a therapeutic effect of tobacco usage but rather that in some instances it is CD rather than UC which develops as a result of influences on the pathogenic processes by smoking.

Abbreviations: IBD, inflammatory bowel disease; $C D$, Crohn's disease; UC, ulcerative colitis; OR, odds ratio; TNBS, trinitrobenzene sulphonic acid; DNBS, dinitrobenzene sulphonic acid. 
Table 1 Description, number, and type of affected sibling pairs in the 242 families studied

\begin{tabular}{lcrrrrr}
\hline \multirow{2}{*}{$\begin{array}{l}\text { No of affected sib } \\
\text { pairs in each family }\end{array}$} & No of families & CD & UC & Mixed & $\begin{array}{l}\text { Total } \\
\text { affected } \\
\text { siblings }\end{array}$ & $\begin{array}{l}\text { Other } \\
\text { affected } \\
\text { relatives }\end{array}$ \\
\cline { 3 - 7 } & 199 & 96 & 59 & 44 & 398 & 112 \\
2 & 4 & 3 & 4 & 1 & 16 & - \\
3 & 32 & 26 & 46 & 24 & 96 & 4 \\
4 & 3 & 8 & 3 & 1 & 15 & - \\
6 & 4 & 15 & 3 & 6 & 16 & 1 \\
Total & 242 & 148 & 115 & 76 & 541 & 117 \\
\hline
\end{tabular}

To arrive at the total number of sib pairs in each affected family, imagine sibs $A, B, C, D$ and $E$; two affected sibs are required for one sib pair, for example, $A B$; three affected siblings make three sib pairs, for example, $A B, B C, A C$; four affected siblings make six sib pairs $A B, A C, A D, B C, B D, C D$; if $D$ and $E$ are the step brother/sister of $A, B$ and $C$ and all are affected, there are four sib pairs $A B, A C, B C, D E$. See also Hauser and colleagues. ${ }^{43}$

$C D$, Crohn's disease; UC, ulcerative colitis.

Theoretically it would be possible to investigate this possibility directly in identical twins discordant both for disease and smoking habit, but such individuals are extremely rare. Consequently, to study the influence of smoking on CD as opposed to UC development, we have considerably enlarged the series of sib pairs discordant for CD/UC and smoking habit, and show that in these closely related individuals it is CD that almost always develops in the smoking sib and UC that develops in the non-smoker. This suggests that in some circumstances smoking can lead to a shift from the UC to the CD phenotype.

\section{PATIENTS AND METHODS}

Between 1991 and 1998, King's College School of Medicine, Guy's Hospital, and St Mark's Hospital, London, coordinated a collaborative programme to identify kindreds with at least two affected siblings with IBD. We identified 242 families with a total of 339 affected sibling pairs by patient interview, postal survey of clinicians, systematic search of hospital records, and advertisement through two UK patient organisations. The 339 affected sib pairs comprised 148 with CD only and 115 with UC. There were 76 sibling pairs where one had CD and the other UC. In the 242 pedigrees, there were a total of 658 affected relatives of which 541 were affected siblings. A breakdown of the structure of these families is shown in table 1.

Clinical details for each of the patients were obtained by personal interview, information acquired from clinicians, and review of hospital records. The diagnosis of IBD and classification as CD and UC were determined by standard diagnostic criteria. ${ }^{28}$ The following information was also collected for each patient: date of birth, sex, age at onset of symptoms, age at diagnosis, presence or absence of extraintestinal disease manifestations, and smoking history (age at which smoking started, number of cigarettes smoked per day, and duration of smoking habit). For patients with CD, additional clinical information included disease localisation (ileal, colonic, or ileocaecal), history of small or large bowel resection, and disease type. The latter was inferred from the dominant clinical and pathological features as defined previously ${ }^{29}$ : in the cicatrising or fibrostenotic form, patients had clinical or radiological evidence of stenosis; in the perforating or fistulising form, patients had evidence of chronic intestinal fistulae or evidence of ileal or colonic perforation. Patients with the inflammatory form of CD had no evidence of stenoses or fistulae. For patients with UC, specific clinical information included extent of disease at the most recent investigation (either pancolitis or distal colitis defined by disease extending proximal or distal to the splenic flexure, respectively) and history of colectomy.

Smoking history was collected by personal interview in the patient's home (by SB, JL, and AM) and divided into two major categories similar to those used by Cottone et al and Lautenbach and colleagues. ${ }^{30} 31$

(A) "Non-smoker" — a patient who had never smoked.

(B) "Ever smoker" - a patient who had smoked at least five cigarettes daily for at least one year of his or her life.

Smoking habit was further subdivided into three groups.

$\left(\mathrm{B}_{1}\right)$ "Smoker at diagnosis" - a patient who had smoked at least five cigarettes daily for at least a year at the time of diagnosis.

$\left(\mathrm{B}_{2}\right)$ "Stopped smoking" — a patient who had given up smoking at least a year before diagnosis.

$\left(\mathrm{B}_{3}\right)$ "Smoker post-diagnosis" — a patient who had only taken up smoking at any time after a diagnosis of IBD had already been established (table 2).

The extent of smoking history was measured in pack years, where 1 pack year constitutes smoking 20 cigarettes a day for one year.

The families were predominantly of European Caucasian origin: 13 families were Jewish, three African, four Asian, and two mixed Caucasian/African. In this familial cohort of patients, smokers were diagnosed with UC at a median age of 31 years, four years older than their non-smoking relatives (median age 27 years).

Study protocols were approved by the ethics committees at the main participating centres and at a further 18 regional hospitals located in London or the South of England. Written informed consent was obtained from all study participants.

\section{Statistical analysis}

Data management and statistical analysis were carried out using SPSS version 7.5 (SPSS Inc., Chicago, Illinois, USA). McNemar's test with Yates' continuity correction was used in the analysis of affected sib pairs discordant for either sex, diagnosis, or smoking habit at diagnosis. Comparisons for age at onset of disease were analysed by the Mann-Whitney test. All affected relatives with familial disease were stratified by diagnosis, sex, and smoking history. $\chi$ tests were used to assess the influence of smoking and sex on the type, site, and extent of disease. A logistic regression model was used to evaluate the independent effects of smoking with estimation of odds ratios (OR) and 95\% confidence intervals (CI) for the relevant outcome variables; these included the presence or absence of the following: $\mathrm{CD}, \mathrm{UC}$, site of disease (ileal, ileocolonic, colonic, pancolitis, distal colitis), subtype of CD (perforating, stenosing, inflammatory), and gastrointestinal resection. These variables were selected for inclusion into the logistic regression model as they were those on which we had comprehensive information, and are standard variables for a study of IBD. While we also had comprehensive information about extraintestinal IBD disease manifestations, these 
Table 2 Comparison of disease type in familial patients with inflammatory bowel disease stratified by smoking habit

\begin{tabular}{lcclc}
\hline & $C D(n(\%))$ & UC (n (\%)) & $\begin{array}{l}\text { OR in favour of CD } \\
(95 \% \mathrm{Cl})\end{array}$ & p Value \\
\hline All patients & 333 & 277 & & \\
Never smoked $(\mathrm{A})$ & $124(37.2)$ & $162(58.5)$ & $0.42(0.3-0.58)^{*}$ & $<0.0001$ \\
Ever smoked $(\mathrm{B})$ & $209(62.8)$ & $115(41.5)$ & $2.37(1.69-3.34)$ & $<0.0001$ \\
Smoked at diagnosis $\left(\mathrm{B}_{1}\right)$ & $180(54.5)$ & $69(24.9)$ & $3.55(2.51-5.02)$ & $<0.0001$ \\
Smoked pre-diagnosis $\left(\mathrm{B}_{2}\right)$ & $10(3)$ & $39(14.1)$ & $0.19(0.09-0.39) \dagger$ & $<0.0001$ \\
Smoked post-diagnosis $\left(\mathrm{B}_{3}\right)$ & $19(5.7)$ & $7(2.5)$ & $2.33(0.97-5.62)$ & 0.053 \\
\hline
\end{tabular}

An odds ratio of greater than 1 favours the development of Crohn's disease (CD), and less than 1 favours the development of ulcerative colitis (UC).

*Favouring UC 2.37 (1.69-3.34)

†Favouring UC 5.05 (2.46-11.41).

$\mathrm{OR}$, odds ratio; $95 \% \mathrm{Cl}, 95 \%$ confidence interval.

occurred too infrequently to be useful in investigating the impact of smoking at diagnosis or at any time before diagnosis. Separate models were used for smoking at diagnosis (yes or no) and whether the patient had ever smoked (yes or no).

\section{RESULTS}

\section{Different effects of smoking in females and males on} the incidence of $C D$ or UC

Comparison of our overall population of familial patients with IBD $(n=658$, of whom 48 were excluded because of incomplete clinical information, females 382 (58\%), mean current age 46.1 years) showed that smoking at diagnosis was positively associated with CD (OR 3.55; 95\% CI 2.50-5.02; $\mathrm{p}<0.001$ ) and inversely with UC (OR 0.28 ; 95\% CI $0.2-0.4$; $\mathrm{p}<0.001)$. However, there was a more pronounced risk of $\mathrm{CD}$ (OR 5.44; 95\% CI 3.33-8.88; $\mathrm{p}=<0.0001$ ) and protection from UC (OR 0.18; 95\% CI 0.11-0.3; p<0.0001) in 151 females smoking at diagnosis compared with 204 female nonsmokers, whereas in 98 male smokers at diagnosis that were compared with 157 male non-smokers, these effects were smaller (smoking related CD risk OR 2.11 ; 95\% CI 1.26-3.52; $\mathrm{p}=0.004$; UC protection in smokers OR $0.47 ; 95 \%$ CI $0.28-0.79)$

\section{Smoking at diagnosis predisposes to the development of $C D$ rather than UC in sib pairs discordant for disease type}

To investigate the effects of smoking on the development of CD and UC in individuals with a similar genetic background, we examined 89 sib pairs with CD or UC who were discordant for smoking at the time of diagnosis (table 3). Of these, $23 \mathrm{sib}$ pairs were also discordant for disease diagnosis, and 21 of these showed UC in the non-smoker and CD in the smoker (OR 10.5; 95\% CI 2.6-92; p<0.0001). In only two sib pairs was

Table 3 Matched analysis of the 89 affected sibling pairs discordant for smoking at diagnosis

\begin{tabular}{lll}
\hline & \multicolumn{2}{l}{ Smokers } \\
\cline { 2 - 3 } & CD sibling & UC sibling \\
\hline Non-smokers & 42 & 2 \\
CD sibling & 21 & 24 \\
UC sibling & 21 \\
\hline
\end{tabular}

23 pairs were discordant for their diagnoses. In 21 of these pairs, the smoker had been diagnosed as having Crohn's disease (CD), and the non-smoker ulcerative colitis (UC) (McNemaras test with Yates' continuity correction $p<0.0001$, odds ratio $10.5,95 \%$ confidence limits 2.6-92; Fisher-Irwin two tailed probability test $p<10^{-8}$ ). There were only two affected sib pairs in which the smoker had developed UC and the non-smoker CD. this situation reversed. Even if we excluded 15 families contributing more than one sib pair, there were 17 sib pairs discordant for both smoking and disease type; of these, 15 had UC in the non-smoker and CD in the smoker (OR 7.5; 95\% CI 1.74-67.6; $\mathrm{p}=0.0036$ ). To examine the possible effects of sex differences in generating discordance, we also looked at 140 sib pairs discordant for sex at diagnosis (table 4); of these, 31 were also discordant for disease diagnosis but here the split was almost even (and not significantly different) between 13 UC females and CD males, and 18 CD females and UC males.

Paired analysis was also undertaken on the $42 \mathrm{CD}$ and 24 UC sib pairs discordant for their smoking habits. For CD, smoking was associated with a greater tendency to develop small bowel disease (OR 3.3; 95\% CI 0.86-18.8; $\mathrm{p}=0.09$ ), and a reduced tendency to develop colonic disease (OR 0.78; $95 \%$ CI $0.43-4.1 ; p=0.6)$. No differences were seen for the influence of smoking on the subtype of CD or extent of UC, although this may reflect the small numbers of patients available for analysis in this group of two way discordant sib pairs. Indeed, in the overall group of familial patients, those who were smokers when diagnosed with CD had increased disease of the fibrostenosing type, and male smokers were predisposed to develop disease in the ileum (table 5).

\section{Threshold for the environmental effect of smoking on the phenotype of IBD}

To examine whether we could detect a lower threshold of cigarette smoking necessary to influence the development of $\mathrm{CD}$, we compared the proportion of patients diagnosed with CD and UC as a function of when they had smoked and the number of pack years (fig 1, table 2). This showed that the increase in susceptibility to CD was associated with a very short smoking history at diagnosis: it was seen in those who had smoked 5 or fewer pack years at diagnosis which was maintained with a more extensive smoking history compared with non-smokers. (A single pack year constitutes smoking 20 cigarettes a day for one year.) However, those patients who gave up smoking at least a year before diagnosis were significantly less likely to develop $\mathrm{CD}$, regardless of the amount they

Table 4 Matched analysis of the 140 affected sibling pairs discordant for sex

\begin{tabular}{lll}
\hline & \multicolumn{2}{l}{ Females } \\
\cline { 2 - 3 } & CD sibling & UC sibling \\
\hline Males & & \\
CD sibling & 56 & 13 \\
UC sibling & 18 & 53 \\
\hline
\end{tabular}

In comparison with smoking discordance, discordance for sex was not significantly associated with the development of either Crohn's disease (CD) or ulcerative colitis (UC) 
Table 5 Influence of smoking and sex on type, site, and extent of familial inflammatory bowel disease.

\begin{tabular}{lll}
\hline & \multicolumn{2}{l}{ Smokers at diagnosis } \\
\cline { 2 - 3 } & Females $(n=151)$ & Males $(n=98)$ \\
\hline$C D$ & $5.44(3.33-8.88)^{* * *} n=122$ & $2.11(1.26-3.52)^{* *} n=58$ \\
Ileal disease & $1.38(0.71-2.71) n=94$ & $3.68(1.42-9.52)^{* *} n=49$ \\
Ileo-caecal disease & $1.86(0.92-3.77) n=99$ & $1.54(0.47-5.02) n=51$ \\
Colonic disease & $1.17(0.66-2.1) n=68$ & $0.27(0.13-0.58)^{* *} n=19$ \\
Fibrostenosing disease & $1.83(1.03-3.24)^{*} n=75$ & $2.49(1.17-5.29)^{*} n=38$ \\
Perforating disease & $0.99(0.51-1.91) n=28$ & $1.07(0.44-2.58) n=12$ \\
Intestinal resection & $2.12(1.17-3.86)^{*} n=96$ & $1.47(0.69-3.13) n=38$ \\
UC & $0.18(0.11-0.3)^{* * *} n=29$ & $0.47(0.28-0.79)^{* *} n=40$ \\
Pancolitis & $1.34(0.59-3.04) n=16$ & $1.21(0.58-2.55) n=20$ \\
Colectomy & $0.95(0.38-2.35) n=8$ & $1.06(0.44-2.59) n=9$ \\
\hline
\end{tabular}

Values are odds ratios $195 \%$ confidence limits)

Patients were stratified by diagnosis, sex, and smoking habit. Female smokers at diagnosis were compared with female non-smoking relatives. Male smokers at diagnosis were compared with male non-smoking relatives. Female smokers were significantly more likely to develop Crohn's disease (CD) $(p<0.001)$, particularly with colonic involvement $(p=0.003)$, and less likely to develop ulcerative colitis (UC) $(p<0.001)$ than male smokers ( ${ }^{*} p<0.05$, $\left.{ }_{* *} \mathrm{p}<0.005,{ }^{* * *} \mathrm{p}<0.0001\right)$.

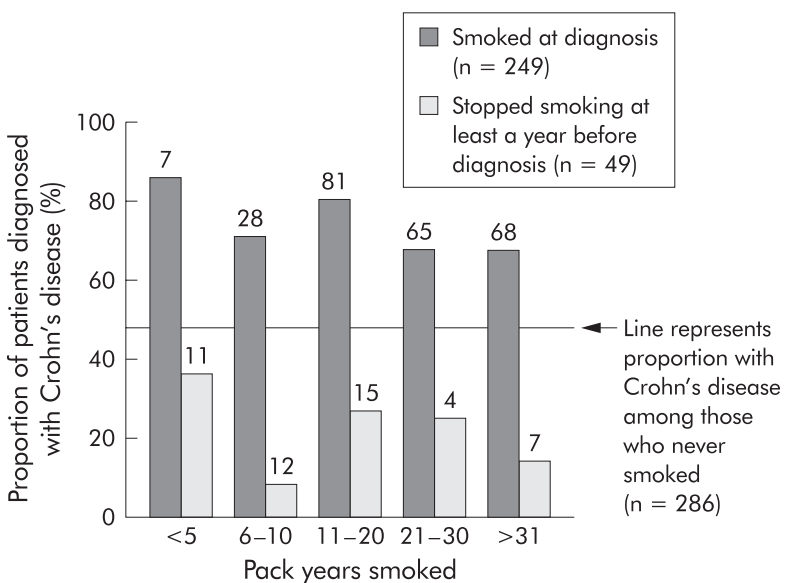

Figure 1 Proportion of patients with familial inflammatory bowel disease diagnosed with Crohn's disease as a function of the cumulative pack years smoked. A pack year is defined as smoking 20 cigarettes a day for one year. Patients who were smokers at diagnosis were compared with former smokers who had given up at least a year before the diagnosis was made. The horizontal line represents the proportion of non-smokers diagnosed with CD (124 of 286 patients; $43.4 \%$ ). Numbers above each bar give the total number of patients in each group from which the proportion with Crohn's disease was derived.

had smoked previously. It was clear that smoking at diagnosis was itself the risk factor for developing CD rather than the cumulative amount smoked prior to diagnosis.

\section{DISCUSSION}

This study confirms that smoking is an important aetiological factor in the pathogenesis of IBD, and that it predisposes to $\mathrm{CD}$, especially in women. However, the existence of sib pairs in which the smoker almost always has CD and the non-smoker UC shows that in some individuals who are closely related smoking can apparently influence whether the phenotype is UC or CD. In this familial cohort, smokers were diagnosed with UC at a median age of four years older than non-smokers, which is also consistent with tobacco usage inhibiting UC and enhancing CD pathogenetic mechanisms.

There are three important caveats in the interpretation that smoking cannot only exacerbate CD and heal UC but also (where the genetic risks are evenly balanced) result in a shift between the two forms. Firstly, while matched analysis of affected sibling pairs has the advantage that it directly compares patients with similar genetic and social susceptibility, our sib pairs were not genetically identical, and therefore gene differences between them could still influence the disease that develops: however, the strength of the effect of smoking in those pairs discordant for disease type and smoking was so strong that it is hard to see how the smoker could almost always have the CD genetic predisposition and the non-smoker the UC genetic predisposition by chance. Secondly, there are many examples of sib pairs discordant for smoking but not disease type (66 of 89 discordant for smoking), and so the effect was only seen in a proportion of cases: in other words, it is likely that genetic risks usually determine whether the disease is CD or UC, and only where these risks are evenly balanced between the two conditions can smoking determine the phenotypic outcome. Thirdly, in discordant sib pairs we could not distinguish whether the mechanism of this effect was due to exacerbation of the CD pathogenesis, protection from the UC pathogenesis, or both. To illustrate this point, one can consider the theoretical situation if enough identical twins were available, when one might be able to examine the strength of these different effects: (i) smoking exacerbates CD (smoker with CD, non-smoker disease free); (ii) smoking protects from UC (smoker disease free, non-smoker UC); (iii) pure shift of phenotype (smoker CD, non-smoker UC). In practice however, understanding the pathogenic mechanisms underlying tobacco usage are likely to come from animal models.

We were unable to detect a lower threshold (below 5 pack years) for the effects of smoking on the development of CD, and indeed it appeared to be effectively reversed regardless of previous exposure of tobacco within a year of giving up smoking. This suggests that the influences of tobacco on disease pathogenesis are on the immunopathogenic mechanisms at disease onset as opposed to the cumulative damage seen for example in atherosclerosis or chronic obstructive airways disease. Studies of the effectiveness of stopping smoking following diagnosis are confounded by the effects of medical intervention, and we cannot reliably assess this.

We found no evidence to indicate that recall bias may have influenced the results of this study. Although it is known that patients can under or over report their cigarette consumption, self reported smoking habit has been shown to exhibit good reliability and validity in cross sectional studies. ${ }^{32}{ }^{33}$ Indeed, compared with affected patients, relatives without IBD who were smokers had a greater cumulative tobacco consumption (in pack years per smoker). While we cannot discount a possible effect of smoking in suppression of colitis symptoms directly, there was no evidence of lead time bias (age of symptomatic onset to age of diagnosis), which was similar in both smokers and non-smokers who developed UC.

It is well recognised that smoking can be associated with development of systemic autoimmune disease, ${ }^{34}$ including 
Goodpasture's syndrome, rheumatoid arthritis, ${ }^{35}{ }^{36}$ and Grave's disease, and increased severity of ophthalmopathy. ${ }^{34}$ The mechanisms underlying the interaction between tobacco consumption and these diseases remain unclear and there are multiple effects of smoking on immune and inflammatory functions, including increased numbers of circulating and tissue neutrophils and lymphocytes and complex alterations of macrophage, $\mathrm{B}$ and $\mathrm{T}$ cell functional responses, depressed natural killer cell function, and increased complement activation. ${ }^{37-39}$ In the oral mucosa, periodontal disease resulting from inflammatory responses against the dental microflora is also exacerbated by smoking. ${ }^{360}$

The effects of cigarette smoke have been evaluated in the rodent model of IBD initiated by intestinal exposure to the haptens trinitrobenzene sulphonic acid (TNBS) or dinitrobenzene sulphonic acid (DNBS). In rats treated with either hapten, passive smoking by the animals exacerbated the resultant disease. Exacerbation of the TNBS effects was found to be associated with increased free radical production and inducible nitric oxide synthase activity ${ }^{41}$ whereas aggravation of DNBS colitis was reversed by hexamethonium, suggesting that a neural pathway is involved. ${ }^{42}$

Chronic IBD is usually divided into CD and UC on the basis of clinical and pathological distinctions, although there are intermediate forms, shared genetic linkages, and families containing members with each condition. We have shown here that in some cases smoking can probably displace the phenotype of chronic IBD from UC to CD-an example of how environmental influences interact with the genetic background to influence not just whether there is disease but also the type of disease that develops. The observation has important implications for studies in the genetic and immunopathological mechanisms of IBD. Finally, the apparent protection that smoking offers against UC may be in part a shift of the cases towards CD.

\section{Authors' affiliations}

S Bridger, I Biarnason, Department of Medicine, Guy's, King's, and St Thomas's School of Medicine and Dentistry, Bessemer Road, London SE5 9PJ, UK

J C W Lee, J E Lennard Jones, St Mark's Hospital, Northwick Park, Harrow, Middlesex HAl 3JU, UK

A J Macpherson, Department of Medicine, Guy's, King's, and St Thomas's School of Medicine and Dentistry, Bessemer Road, London SE5 9PJ, UK, and Institute of Experimental Immunology and Abteilung für Gastroenterologie, University Hospital Zürich, Schmelzbergstrasse 12, $\mathrm{CH}-8091$ Zurich, Switzerland

\section{REFERENCES}

1 Podolsky DK. Inflammatory bowel disease (1). N Engl J Med 1991;325:928-37.

2 Fiocchi C. Inflammatory bowel disease: etiology and pathogenesis. Gastroenterology 1998;115:182-205.

3 Papadakis KA, Targan SR. Role of cytokines in the pathogenesis of inflammatory bowel disease. Annu Rev Med 2000;51:289-98.

4 MacDonald TT, Monteleone G, Pender SL. Recent developments in the immunology of inflammatory bowel disease. Scand J Immunol 2000;51:2-9.

5 Duerr RH, Barmada MM, Zhang L, et al. High-density genome scan in Crohn disease shows confirmed linkage to chromosome 14q11-12. Am J Hum Genet 2000;66:1857-62.

6 Rioux JD, Silverberg MS, Daly M, et al. Genomewide search in Canadian families with inflammatory bowel disease reveals two novel susceptibility Loci. Am J Hum Genet 2000;66:1863-70.

7 Brant SR, Fu Y, Fields CT, et al. American families with Crohn's disease have strong evidence for linkage to chromosome 16 but not chromosome 12. Gastroenterology 1998;115:1056-61.

8 Parkes M, Satsangi J, Lathrop GM, et al. Susceptibility loci in inflammatory bowel disease. Lancet 1996;348:1588.

9 Cho JH, Nicolae DL, Ramos R, et al. Linkage and linkage disequilibrium in chromosome band $1 \mathrm{p} 36$ in American Chaldeans with inflammatory bowel disease. Hum Mol Genet 2000;9: 1425-32.

10 Annese V, Latiano A, Bovio P, et al. Genetic analysis in Italian families with inflammatory bowel disease supports linkage to the IBDI locus-a GISC study. Eur J Hum Genet 1999;7:567-73.

11 Hampe J, Schreiber S, Shaw SH, et al. A genomewide analysis provides evidence for novel linkages in inflammatory bowel disease in a large European cohort. Am J Hum Genet 1999;64:808-16.
12 Hampe J, Shaw SH, Saiz R, et al. Linkage of inflammatory bowel disease to human chromosome 6p. Am J Hum Genet 1999;65:1647-55.

13 Yang H, Plevy SE, Taylor K, et al. Linkage of Crohn's disease to the major histocompatibility complex region is detected by multiple non-parametric analyses. Gut 1999:44:519-26.

14 Curran ME, Lau KF, Hampe J, et al. Genetic analysis of inflammatory bowel disease in a large European cohort supports linkage to chromosomes 12 and 16. Gastroenterology 1998;115:1066-71.

15 Cavanaugh JA, Callen DF, Wilson SR, et al. Analysis of Australian Crohn's disease pedigrees refines the localization for susceptibility to inflammatory bowel disease on chromosome 16. Ann Hum Genet 1998;62:291-8.

16 Ma Y, Ohmen JD, Li Z, et al. A genome-wide search identifies potential new susceptibility loci for Crohn's disease. Inflamm Bowel Dis 1999;5:271-8.

17 Duerr RH, Barmada MM, Zhang L, et al. Linkage and association between inflammatory bowel disease and a locus on chromosome 12. Am J Hum Genet 1998;63:95-100.

18 Hugot JP, Laurent-Puig P, Gower-Rousseau C, et al. Mapping of a susceptibility locus for Crohn's disease on chromosome 16. Nature 1996:379:821-3.

19 Satsangi J, Parkes M, Louis E, et al. Two stage genome-wide search in inflammatory bowel disease provides evidence for susceptibility loci on chromosomes 3, 7 and 12. Nat Genet 1996;14:199-202.

20 Ohmen JD, Yang HY, Yamamoto KK, et al. Susceptibility locus for inflammatory bowel disease on chromosome 16 has a role in Crohn's disease, but not in ulcerative colitis. Hum Mol Genet 1996;5:1679-83.

21 Cho JH, Nicolae DL, Gold LH, et al. Identification of novel susceptibility loci for inflammatory bowel disease on chromosomes $1 \mathrm{p}, 3 \mathrm{q}$, and $4 \mathrm{q}$ : evidence for epistasis between $1 \mathrm{p}$ and IBD 1. Proc Natl Acad Sci USA 1998;95:7502-7.

22 Mirza MM, Lee J, Teare D, et al. Evidence of linkage of the inflammatory bowel disease susceptibility locus on chromosome 16 (IBD1) to ulcerative colitis. J Med Genet 1998:35:218-21.

23 Ogura $Y$, Bonen DK, Inohara N, et al. A frameshift mutation in NOD2 associated with susceptibility to Crohn's disease. Nature 2001:411:603-6.

24 Hugot JP, Chamaillard M, Zouali $\mathrm{H}$, et al. Association of NOD2 leucine-rich repeat variants with susceptibility to Crohn's disease. Nature 2001;411:599-603.

25 Hampe J, Cuthbert A, Croucher PJ, et al. Association between insertion mutation in NOD2 gene and Crohn's disease in German and British populations. Lancet 2001;357:1925-8.

26 Calkins BM. A meta-analysis of the role of smoking in inflammatory bowel disease. Dig Dis Sci 1989;34:1841-54.

27 Tobin MV, Logan RF, Langman M, et al. Cigarette smoking and inflammatory bowel disease. Gastroenterology 1987;93:316-21

28 Lee JC, Lennard-Jones JE. Inflammatory bowel disease in 67 families each with three or more affected first-degree relatives. Gastroenterology 1996;111:587-96.

29 Grandbastien B, Peeters M, Franchimont D, et al. Anticipation in familial Crohn's disease. Gut 1998;42:170-4

30 Cottone $M$, Rosselli $M$, Orlando A, et al. Smoking habits and recurrence in Crohn's disease. Gastroenterology 1994;106:643-8.

31 Lautenbach E, Berlin JA, Lichtenstein GR. Risk factors for early postoperative recurrence of Crohn's disease. Gastroenterology 1998; 115:259-67

32 Slattery ML, Hunt SC, French TK, et al. Validity of cigarette smoking habits in three epidemiologic studies in Utah. Prev Med 1989;18:1 1-19.

33 Patrick DL, Cheadle A, Thompson DC, et al. The validity of self-reported smoking: a review and meta-analysis. Am J Public Health 1994:84:1086-93.

34 Prummel MF, Wiersinga WM. Smoking and risk of Graves' disease. JAMA 1993;269:479-82

35 Saag KG, Cerhan JR, Kolluri S, et al. Cigarette smoking and rheumatoid arthritis severity. Ann Rheum Dis 1997:56:463-9.

36 Barbour SE, Nakashima K, Zhang JB, et al. Tobacco and smoking: environmental factors that modify the host response (immune system) and have an impact on periodontal health. Crit Rev Oral Biol Med 1997:8:437-60

37 Holt PG. Immune and inflammatory function in cigarette smokers. Thorax 1987;42:241-9.

38 Francus T, Klein RF, Staiano-Coico L, et al. Effects of tobacco glycoprotein (TGP) on the immune system. II. TGP stimulates the proliferation of human T cells and the differentiation of human B cells into Ig secreting cells. J Immunol 1988;140:1823-9.

39 George J, Levy Y, Shoenfeld Y. Smoking and immunity: an additional player in the mosaic of autoimmunity. Scand J Immunol 1997;45:1-6.

40 Schenkein HA, Gunsolley JC, Koertge TE, et al. Smoking and its effects on early-onset periodontitis. J Am Dent Assoc 1995;126:1 107-13.

41 Guo X, Wang WP, Ko JK, et al. Involvement of neutrophils and free radicals in the potentiating effects of passive cigarette smoking on inflammatory bowel disease in rats. Gastroenterology 1999:117:884-92.

42 Galeazzi F, Blennerhassett PA, Qiu B, et al. Cigarette smoke aggravates experimental colitis in rats. Gastroenterology 1999;1 17:877-83.

43 Hauser ER, Boehnke M, Guo SW, et al. Affected-sib-pair interval mapping and exclusion for complex genetic traits: sampling considerations. Genet Epidemiol 1996;13:117-37. 